\title{
Changes in brain connectivity related to the treatment of depression measured through fMRI: a systematic review
}

\author{
Esteve Gudayol-Ferré ${ }^{1 *}$, Maribel Peró-Cebollero ${ }^{2}$, Andrés A. González-Garrido ${ }^{3}$ and \\ Joan Guàrdia-Olmos ${ }^{2}$
}

${ }^{1}$ Facultad de Psicología, Universidad Michoacana de San Nicolás de Hidalgo, Morelia, Mexico, ${ }^{2}$ Departament de Metodologia de les Ciències del Comportament, Facultat de Psicologia, Institut de Recerca en Cervell, Cognició i Conducta IR3C, Universitat de Barcelona, Barcelona, Spain, ${ }^{3}$ Instituto de Neurociencias, Universidad de Guadalajara, Guadalajara, Mexico

OPEN ACCESS

Edited by:

Lutz Jäncke,

University of Zurich, Switzerland

Reviewed by:

Xin Di,

New Jersey Institute of Technology, USA

Uwe Herwig,

University Hospital for Psychiatry,

Switzerland

*Correspondence:

Esteve Gudayol-Ferré

ferre@umich.mx

Received: 06 July 2015 Accepted: 06 October 2015 Published: 03 November 2015

Citation:

Gudayol-Ferré E, Peró-Cebollero M, González-Garrido AA and

Guàrdia-Olmos J (2015) Changes in brain connectivity related to the treatment of depression measured through fMRI: a systematic review.

Front. Hum. Neurosci. 9:582.

doi: 10.3389/fnhum.2015.00582
Depression is a mental illness that presents alterations in brain connectivity in the Default Mode Network (DMN), the Affective Network (AN) and other cortical-limbic networks, and the Cognitive Control Network (CCN), among others. In recent years the interest in the possible effect of the different antidepressant treatments on functional connectivity has increased substantially. The goal of this paper is to conduct a systematic review of the studies on the relationship between the treatment of depression and brain connectivity. Nineteen studies were found in a systematic review on this topic. In all of them, there was improvement of the clinical symptoms after antidepressant treatment. In 18 out of the 19 studies, clinical improvement was associated to changes in brain connectivity. It seems that both DMN and the connectivity between cortical and limbic structures consistently changes after antidepressant treatment. However, the current evidence does not allow us to assure that the treatment of depression leads to changes in the $\mathrm{CCN}$. In this regard, some papers report a positive correlation between changes in brain connectivity and improvement of depressive symptomatology, particularly when they measure cortical-limbic connectivity, whereas the changes in DMN do not significantly correlate with clinical improvement. Finally, some papers suggest that changes in connectivity after antidepressant treatment might be partly related to the mechanisms of action of the treatment administered. This effect has been observed in two studies with stimulation treatment (one with rTMS and one with ECT), and in two papers that administered three different pharmacological treatments. Our review allows us to make a series of recommendations that might guide future researchers exploring the effect of anti-depression treatments on brain connectivity.

Keywords: depression, depression treatment, brain connectivity, fMRI, antidepressants

\section{INTRODUCTION}

Major Depressive Disorder (MDD) and other depressive mood disorders present numerous structural and functional alterations of the encephalon associated both to the physiopathology of depression and its diverse symptomatic manifestations (Rogers et al., 2004; Fitzgerald et al., 2014; Wise et al., 2014). Thus, depressive syndromes have been consistently related to reductions in brain 
volume in several areas related to the regulation of mood such as the anterior cingulate cortex (ACC), along with certain regions from the medial prefrontal cortex (mPFC), e.g., the orbitofrontal cortex (OFC) and the ventromedial cortex (vmPFC). Reductions have also been found in the volume of the lateral prefrontal cortex (LPFC), the basal ganglia, and the hippocampus, among other structures (Wise et al., 2014).

Likewise, functional Magnetic Resonance Imaging (fMRI) allowed us to spot differences in the activation of these and other brain areas, so that, in depressive symptoms, we findrather consistently-an increase in the activation of the mPFC, the amygdala, and the hippocampus in depressed subjects with respect to the control subjects (Rose et al., 2006; Siegle et al., 2007; Wise et al., 2014). It is likewise common for depressed patients to show fewer activations than healthy persons on the LPFC, the inferior parietal lobe (BA 40), the posterior cingulate cortex (PCC), and the striatum, among other structures (Rose et al., 2006; Siegle et al., 2007; Wise et al., 2014).

Most fMRIs on depression compare the activation found in the cerebral regions above mentioned, among others, to that observed in healthy persons (Chen et al., 2008). However, in recent years, the studies on functional and effective connectivity have revealed that the several brain structures related to behavior do not work in isolation, but they form complex functional integration networks (Friston, 2011). Thus, the brain structures altered in depression are part, in turn, of several connectivity networks. Although the different papers on connectivity in depression sometimes show contradictory results, most of the evidence in the literature suggests that, in MDD, connectivity networks at rest and the connectivity networks activated during specific tasks are all altered (Wang et al., 2012). Accordingly, affective disorders have been linked to alterations of the Default Mode Network (DMN), the Affective Network (AN), the Salience Network (SN), and the Cognitive Control Network (CCN), among others (Dutta et al., 2014).

DMN is a network that becomes active when we are not conducting complex cognitive tasks. DMN includes frontal areas such as the ventromedial prefrontal cortex (vmPFC) and portions of the anterior cingulate cortex (ACC), and from the orbitofrontal cortex (OFC) and parietal areas like the posterior cingulate cortex (PCC) and the medial, lateral, and inferior parietal cortex (Raichle et al., 2001; Greicius et al., 2003). It has been linked to self-reference processes and their alteration; while the pathological interactions of DMN with other networks such as the SN and the CCN would be linked to the states of pathological rumination frequently presented by depressed patients (Broyd et al., 2009; Belleau et al., 2014; Jacobs et al., 2014). It is undoubtedly the most widely studied network in mood disorders and the studies generally show that DMN is hyperactivated in depressed patients (Greicius et al., 2007; Liston et al., 2014). Nevertheless, some papers report the opposite pattern in MDD (Veer et al., 2010; see Wang et al., 2012 for a review), and some studies report hyperactivation and hypoactivation patterns at the same time between different structures within DMN (Wu et al., 2011).

The AN is formed by connections between the ACC, the amygdala, the hypothalamus, and other limbic structures. It has also been reported as altered in MDD (Sheline et al., 2010; Salomons et al., 2014), and since its alterations are involved in hunger, sleep, and sexual conduct, it has been related to the presence of vegetative symptoms in depression (Sheline et al., 2010). It has also been found that depression could present patterns of hypoconnectivity between cortical-limbic structures when the brain is actively processing affective information (Anand et al., 2005; Chen et al., 2008).

The CCN is formed by frontal areas, specifically the dorsolateral prefrontal cortex (DLPFC) and the dorsal anterior cingulate cortex (dACC), and parietal posterior areas, mostly the posterior cingulate cortex (PCC). It is involved in the top-down or goal-directed regulation of attention and in the regulation of working-memory (Corbetta and Shulman, 2002) and it also includes medial temporal lobe parts (Greicius et al., 2009). This network becomes impaired in depression (Rogers et al., 2004; Fales et al., 2008). Some studies show that depressed patients present less connectivity in this network than control subjects (Aizenstein et al., 2009; Liston et al., 2014).

Further evidence suggests that, in depressive disorders, there are alterations of functional connectivity at rest between structures that are part of different networks, as well as in coupling them. For example, the alteration of connectivity between cortical-limbic structures such as the connection between the amygdala and ACC, and the amygdala and DLPFC, among others, has been related to the cognitive biases and neuropsychological alterations of depression (Thomas and Elliott, 2009). It has likewise been suggested that the hyperconnectivity of $\mathrm{DMN}$ and the $\mathrm{SN}$ with $\mathrm{CCN}$ is related to rumination in depression (Jacobs et al., 2014). In addition to the studies of the networks at rest in patients with MDD, further anomalies have been described in brain connectivity, such as alterations in cerebellum-brain connectivity in depressed patients (Guo et al., 2013), or alterations in the interhemispheric functional coordination related to disturbances in the connectivity between both cerebral hemispheres (Wei et al., 2014). Likewise, alterations of the connectivity in the affective network (AN) have been reported in depressed patients, as well as between cortical-limbic structures in emotional processing paradigms in depression (Anand et al., 2005; Chen et al., 2008; Delaveau et al., 2011). In addition, a recent metaanalysis by Kaiser et al. (2015) included 27 seed-based voxelwise connectivity studies in MDD. That study confirmed the alterations in the connectivity of both rest networks and in network coupling. More specifically, it concluded that this illness is characterized by several connectivity alterations in both networks and in network coupling. More specifically, patients with MDD presented hypoconnectivity within the frontoparietal network, hypoconnectivity between frontoparietal systems and parietal areas of the dorsal attention network, hyperconnectivity within the DMN, and hyperconnectivity between frontoparietal control areas and regions of the default network (Kaiser et al., 2015). The authors also provided a model in which the widespread network dysfunction underlies core affective and cognitive alterations in MDD (Kaiser et al., 2015).

Given the generalized alterations of brain connectivity in depression, in recent years there has been substantially increasing 
interest in the possible effect of the different antidepressive treatments on functional connectivity in this illness, although this phenomenon has been much less studied. Studies on functional neuroimaging show that antidepressive treatment is capable of normalizing brain activations in depressed patients during affective tasks in areas such as DLPFC, Dorsomedial Prefrontal Cortex (DMPFC), and ventrolateral Prefrontal Cortex (VLPFC), among others (Delaveau et al., 2011). Moreover, a recent metaanalysis by Ma (2014) studied the effects of antidepressants on brain activity underlying emotional processing. Their results showed that antidepressant treatments had effects on the activation of limbic core structures such as the amygdala, the thalamus, and ACC, and in other emotional processing structures like MPFC, the insula, and the putamen. Antidepressant medication increased the activity of these structures when the subjects processed positive emotions, whereas the same medication decreased the activity of the same structures while processing negative emotions. For these reasons, it is perfectly plausible to expect that, after the same treatments, the depressed patients will present changes in connectivity related to illness improvement. Accordingly, there is increasing interest in knowing the effect of antidepressants and other somatic treatments for depression on brain connectivity.

The goal of this review is to conduct a systematic review of the studies focusing on the relationship between the treatment of depression and brain connectivity to try to identify some common patterns. Accordingly, we will review the changes in brain connectivity that occur in DMN and CCN after antidepressant treatment, and the changes among other corticallimbic connections in studies at rest and in studies of activation during emotional tasks. Likewise, we will review some papers measuring changes in connectivity in other brain structures. Additionally, we will review, where possible, the relationship between changes in brain connectivity and clinical improvement observed after antidepressive treatment. To conclude, and also where possible, we will review the possible "causal" relationships between the type of treatment administered and the specific connectivity changes observed when using a particular treatment in MDD.

\section{MATERIALS AND METHODS}

\section{Article Selection}

The articles were selected after a search in the following databases: Pubmed, Psychinfo, and Google Scholar. To locate the papers, the following keywords were searched: "depression treatment," "connectivity," and "fMRI" or "functional Magnetic Resonance Imaging." The term "depression treatment" was replaced by "unipolar depression treatment" and "major depressive disorder treatment" and the previous terms were combined with the Boolean link "and" in several bibliographical searches. Such process was conducted by two independent researchers who reproduced the paper selection process. The initial level of agreement was of $98 \%$ and they concurred the decision to include or not they articles on which they disagreed.
That same term was also replaced by all the existing second-generation antidepressants, and each antidepressant was combined with the other keywords. Out of all the articles recovered, their suitability was assessed for the goals of the current paper on the base of the selection criteria below. The studies had to contain a sample of patients with one or more active depressive syndromes that had been treated with at least one standard depression treatment: antidepressants, Electroconvulsive Therapy (ECT), or psychotherapy. We also included those treated with the experimental treatment repetitive Transcranial Magnetic Stimulation (rTMS), due to its increasing use. The papers had to take, at least, two fMRI measures in the group of depressed patients, one before and the other after finishing the antidepressive treatment. Under these criteria, and in an early stage, 92 different articles were found in the different databases, once duplicities had been spotted in each database. Out of the articles identified, we deleted those not bearing empirical evidence on one connectivity network linked to MDD. Thirty-six papers were then discarded $(39.13 \%)$ as they mentioned the topic tangentially. Out of the remaining papers (56), we deleted those that only presented the correlations matrices but not specific connectivity models, given their strictly descriptive goal, regardless of considerations on functional or structural connectivity. Under this criterion, we deleted 26 further papers $(28.26 \%)$. We analyzed the remaining 30 individually, and we discarded those not offering all the information on the connectivity model, or where such model was partial. This way, we deleted 10 more papers $(10.86 \%$ of the total), and thus there remained 20 papers suitable for analysis. Eventually, one of them was discarded because the connectivity data were duplicated in a previous publication. Accordingly, there remained a total of 19 articles measuring changes in brain connectivity in depressed patients before and after treatment. The following flow chart shows this selection process of articles analyzed, which carry the symbol ${ }^{*}$ in the bibliography (Figure 1).

Once the selection process was finished, both researchers conducted the selection of information from each paper and obtained, eventually, a $100 \%$ agreement on the data of each paper analyzed.

\section{RESULTS}

After searching and applying the criteria, we found 19 articles measuring changes in brain connectivity associated to antidepressive response (Anand et al., 2005; Chen et al., 2008; Aizenstein et al., 2009; Lisiecka et al., 2011; Wu et al., 2011; Beall et al., 2012; Perrin et al., 2012; Abbott et al., 2013, 2014; Andreescu et al., 2013; Heller et al., 2013; Li et al., 2013; Posner et al., 2013; Baeken et al., 2014; Liston et al., 2014; Salomons et al., 2014; Wang et al., 2014; Wei et al., 2014; Yang et al., 2014).

\section{Clinical Results}

Out of the 19 studies analyzed, 11 were conducted on patients with MDD (Anand et al., 2005; Chen et al., 2008; Lisiecka et al., 2011; Beall et al., 2012; Abbott et al., 2013, 2014; Heller et al., 2013; Li et al., 2013; Liston et al., 2014; Salomons et al., 2014; 


\section{2 records identified in Pubmed}

86 records identified in Psyclnfo and Google Scholar.

Keywords: "unipolar depression treatment" \&

"functional Magnetic Resonance Imaging" or fMRI

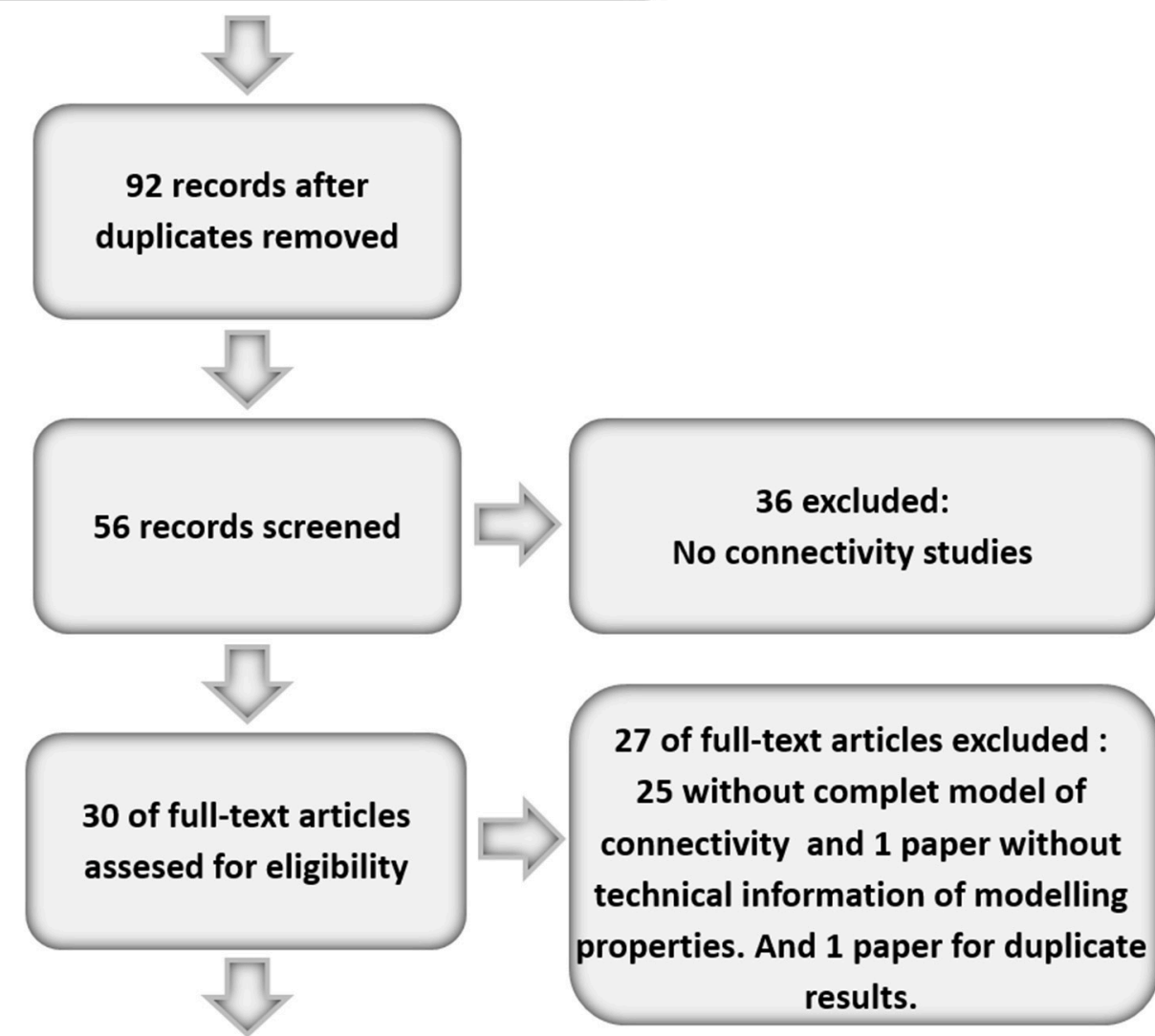

19 studies included

in the descriptive

analysis.

FIGURE 1 | Flow chart of the paper selection process.

Wang et al., 2014). Three studies were conducted on patients with a MDD diagnosis, but in the Late-onset Depression variant (LOD) (Aizenstein et al., 2009; Wu et al., 2011; Andreescu et al., 2013). One was conducted on dysthymic patients (Posner et al., 2013), one on patients with a "unipolar depression" diagnosis (Yang et al., 2014), two on patients with a "severe depression" diagnosis (Perrin et al., 2012; Baeken et al., 2014), and one on patients with "diagnosis of depression" (Wei et al., 2014). None of the studies were conducted on depressive-stage bipolar patients although one study included 4 patients with that diagnosis in its 21-participant sample (Liston et al., 2014), and another included an unspecified number of bipolar patients out of a total of 6 participants (Beall et al., 2012).
With regard to the treatments administered, 11 studies administered antidepressant treatments (Anand et al., 2005; Chen et al., 2008; Aizenstein et al., 2009; Lisiecka et al., 2011; Wu et al., 2011; Andreescu et al., 2013; Heller et al., 2013; Li et al., 2013; Posner et al., 2013; Wang et al., 2014; Yang et al., 2014). The duration of the treatments varied, ranging from 4 weeks (Lisiecka et al., 2011) to 12 weeks (Aizenstein et al., 2009; Andreescu et al., 2013; Li et al., 2013). In the 11 studies where an antidepressant treatment was administered, it was successful, so the depressive symptoms were reduced or remitted in the majority of the patients. This and further relevant clinical information has been summarized in Table 1. 


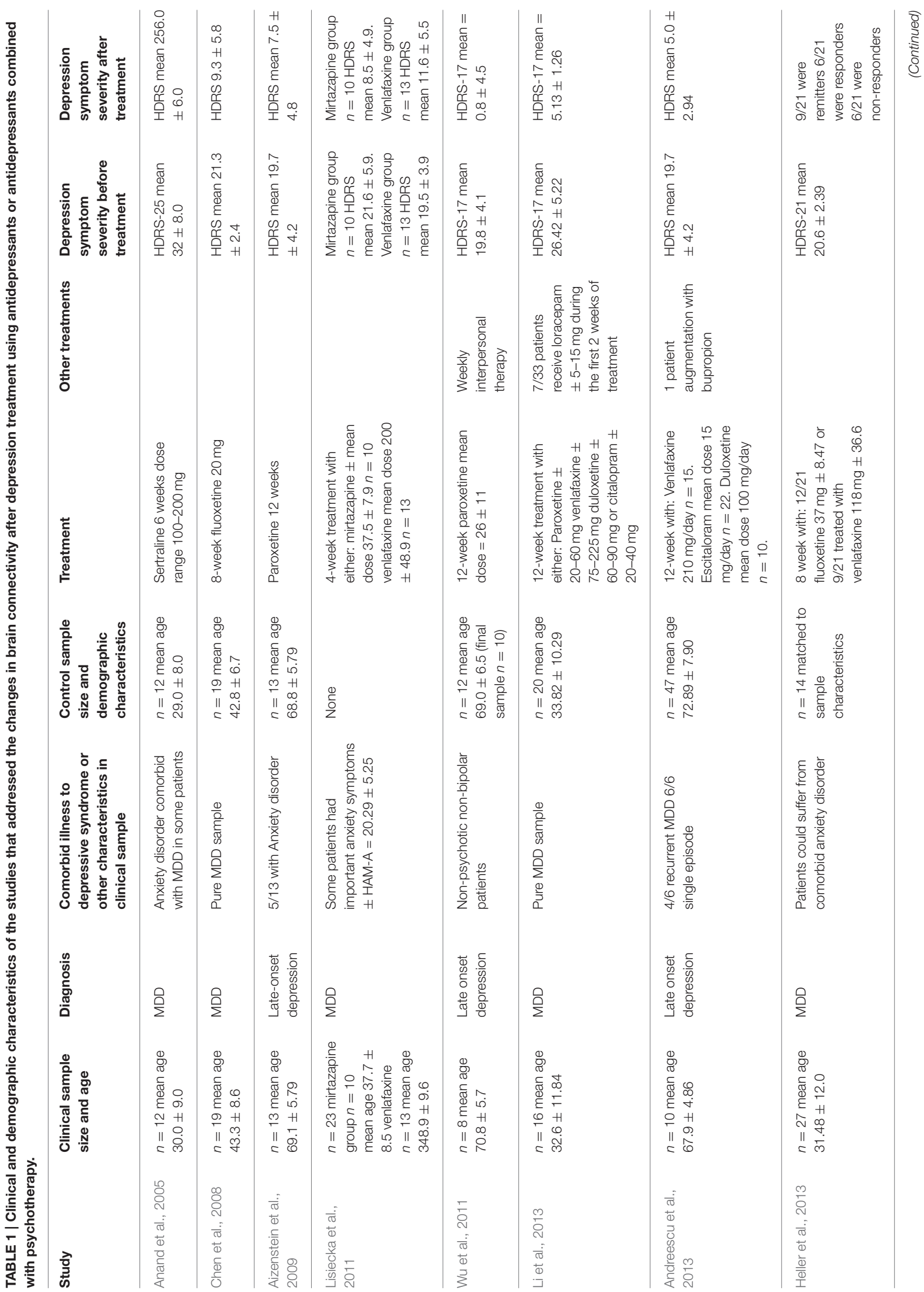




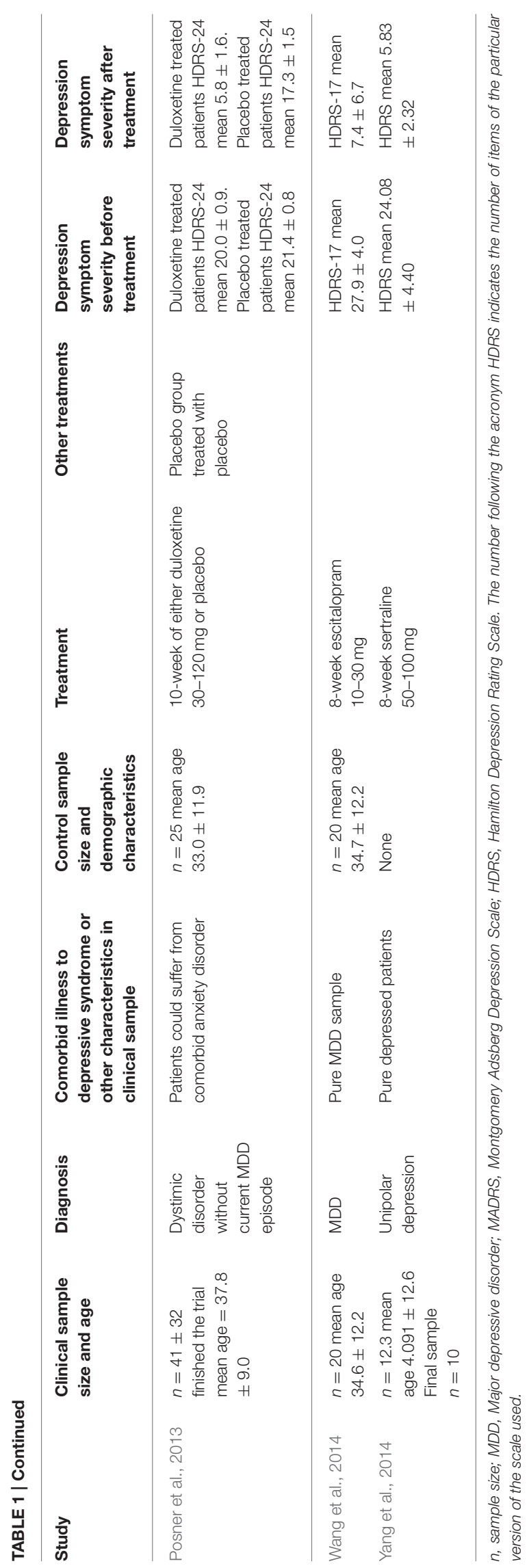

Out of the 18 papers analyzed, 5 of them studied connectivity after ECT (Beall et al., 2012; Perrin et al., 2012; Abbott et al., 2013, 2014; Wei et al., 2014). The treatment was administered in a number of sessions ranging from an average of 7 (Wei et al., 2014) to 11 (Abbott et al., 2013, 2014). All the papers with low numbers show that the treatment was administered efficiently, and that the response or remission of depressive symptoms was achieved in the majority of patients. Three papers used rTMS as a depression treatment (Baeken et al., 2014; Liston et al., 2014; Salomons et al., 2014). In these papers, 25 and 20 rTMS sessions were administered, respectively. All the papers obtained a response or a partial response to such antidepressive therapy. This and further relevant clinical information has been summarized in Table 2.

\section{Connectivity Results}

Out of the 18 studies, 12 had a "longitudinal case-control study" design (Anand et al., 2005; Chen et al., 2008; Aizenstein et al., 2009; Wu et al., 2011; Abbott et al., 2013, 2014; Andreescu et al., 2013; Heller et al., 2013; Li et al., 2013; Liston et al., 2014; Wang et al., 2014; Wei et al., 2014). Out of these, however, the paper by Heller et al. (2013) reports pre-post results only for the behavioral data. One paper had a Mixed-factorial design with two treatment groups (Lisiecka et al., 2011). One combined a mixed factorial design with placebo-controlled clinical trial methodologies (Posner et al., 2013), and one had a shamcontrolled cross-over design (Baeken et al., 2014). The remaining works were conducted with a pre- and post-treatment design (Beall et al., 2012; Perrin et al., 2012; Salomons et al., 2014; Yang et al., 2014). According to the type of paradigm, out of the 11 papers that administered antidepressant treatments, 6 assessed connectivity at rest (Wu et al., 2011; Andreescu et al., 2013; Li et al., 2013; Posner et al., 2013; Wang et al., 2014; Yang et al., 2014), 4 assessed brain connectivity using different activation paradigms (Chen et al., 2008; Aizenstein et al., 2009; Lisiecka et al., 2011; Heller et al., 2013), and one work used both approaches (Anand et al., 2005). Out of the 8 works that treated patients with somatic non-pharmacological treatments, 7 were performed at rest (Perrin et al., 2012; Abbott et al., 2013, 2014; Baeken et al., 2014; Liston et al., 2014; Salomons et al., 2014; Wei et al., 2014) and one paper used emotional and cognitive paradigms and resting measures (Beall et al., 2012).

These and further methodological characteristics have been summarized in Tables 3A,B, 4A,B.

As regards the studies' results on connectivity, seven studies approached the effect of depression treatment on DMN connectivity (Wu et al., 2011; Beall et al., 2012; Abbott et al., 2013; Andreescu et al., 2013; Li et al., 2013; Posner et al., 2013; Liston et al., 2014). Four studies (Andreescu et al., 2013; Li et al., 2013; Posner et al., 2013; Liston et al., 2014) found DMN hyperconnectivity in depressed patients with respect to the control subjects. In all of them, connectivity reductions were observed in DMN after the treatment (Li et al., 2013; Posner et al., 2013; Liston et al., 2014), except for the paper by Andreescu et al. (2013), where-somewhat paradoxicallyit was associated to an increased connectivity in the anterior, 


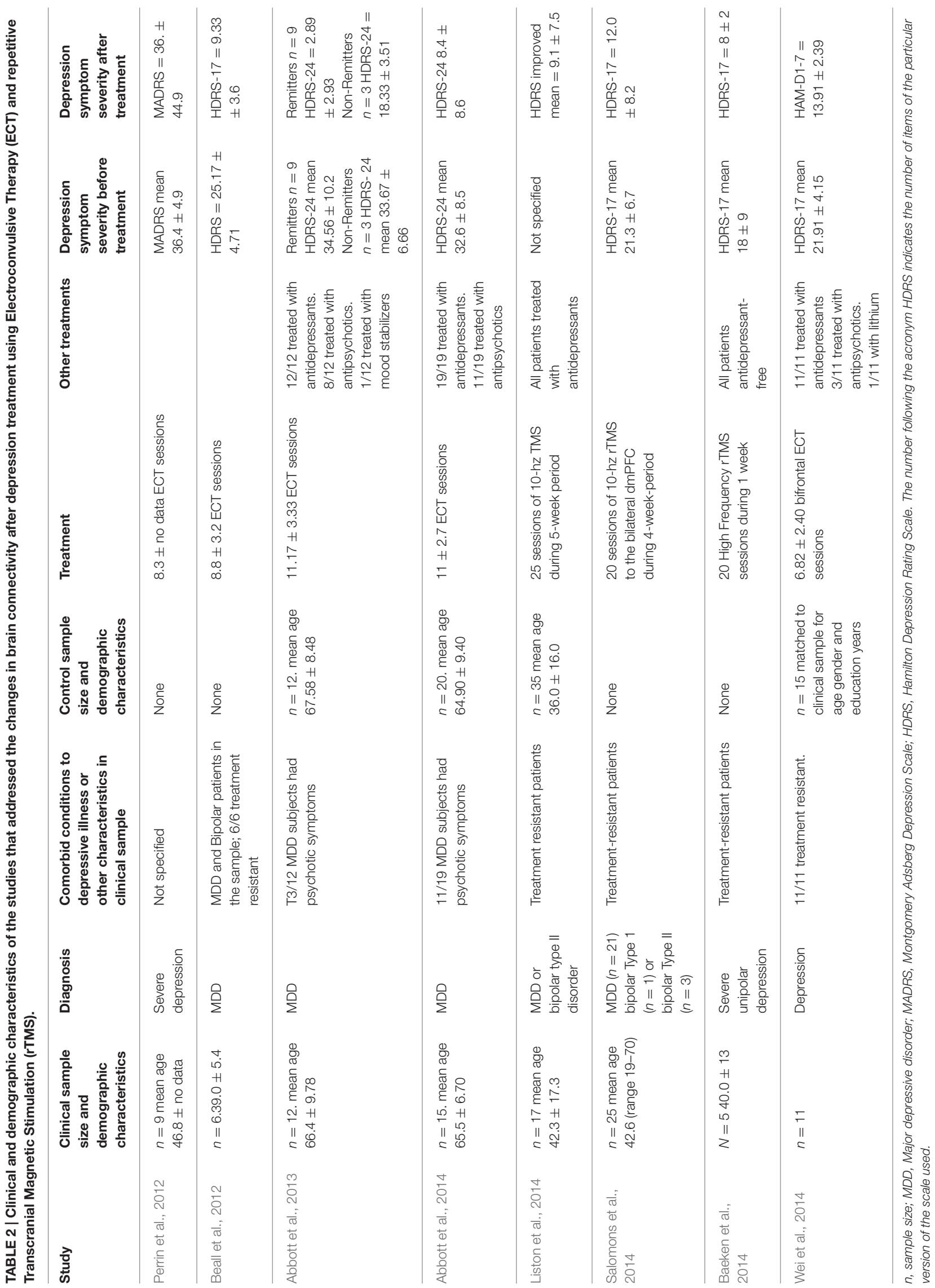




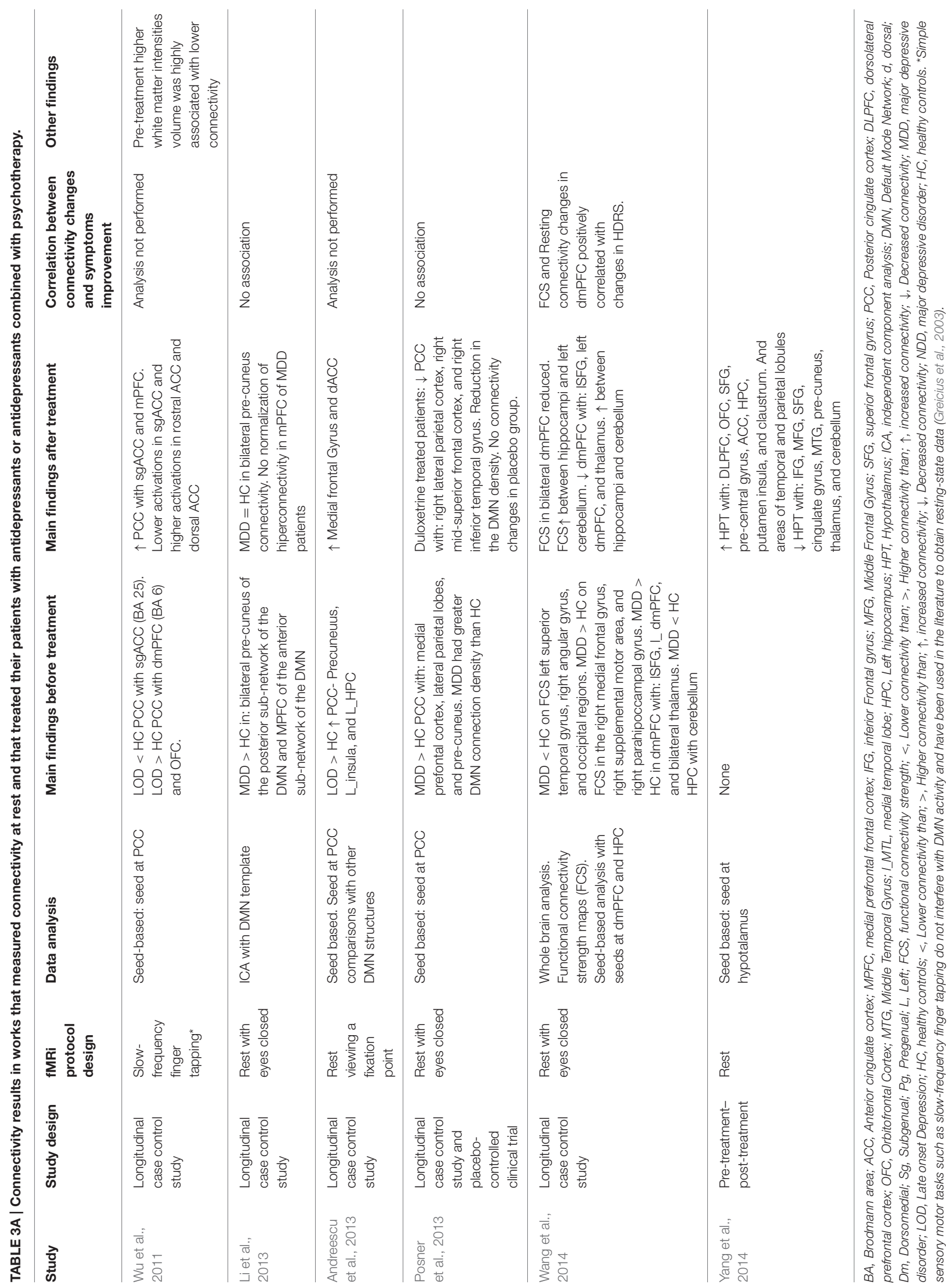




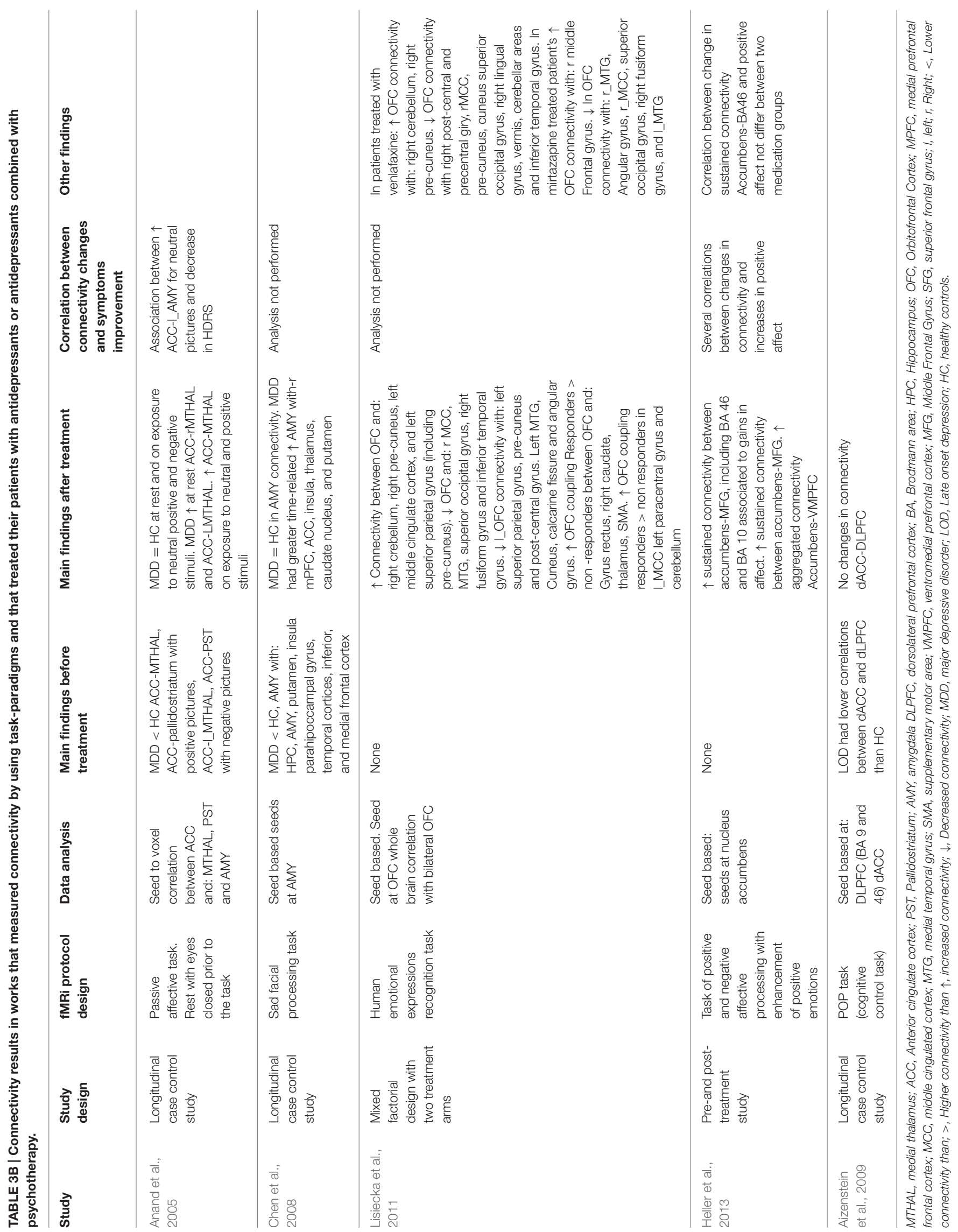




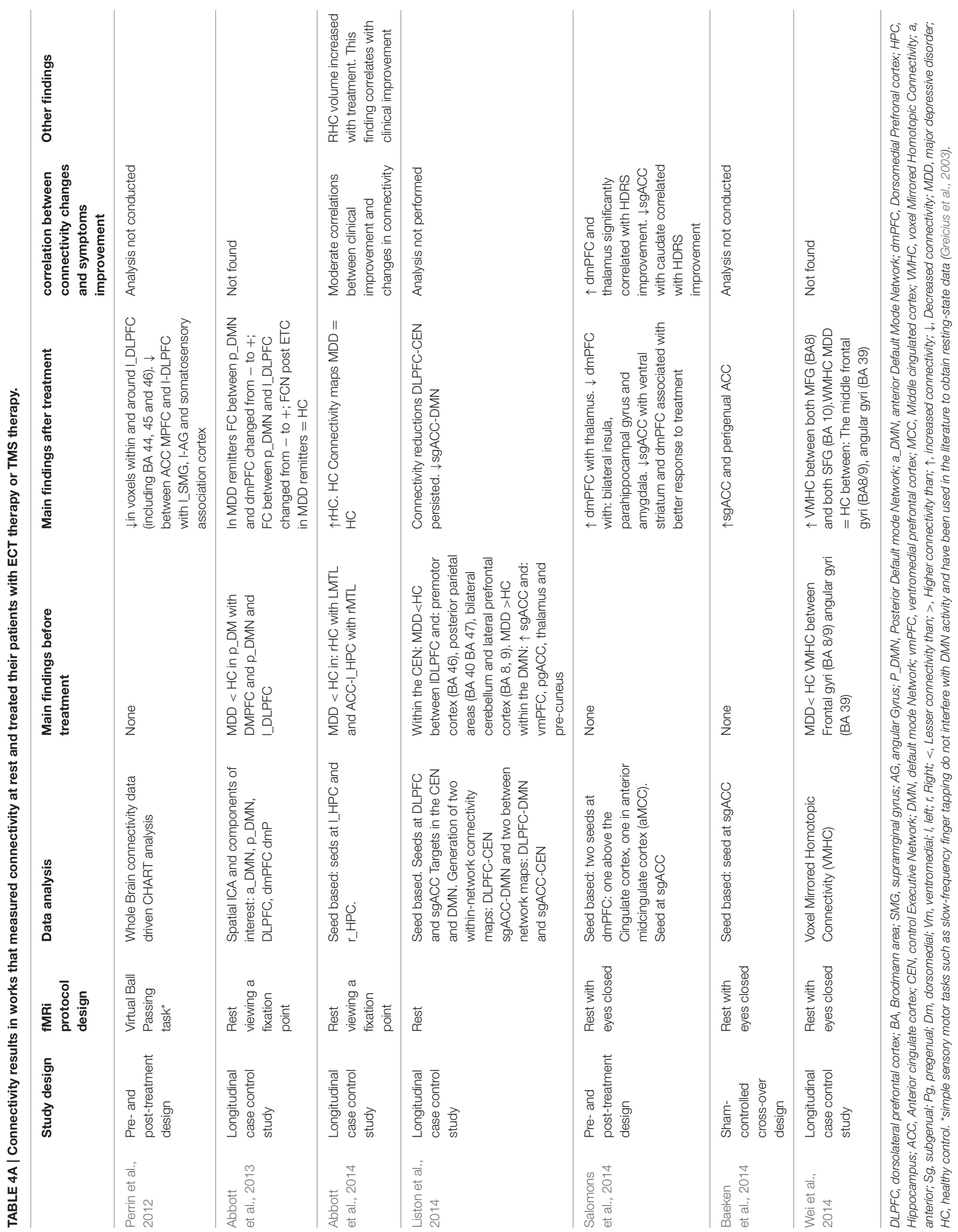


frontal nodes of the DMN, in which such "frontalization" is interpreted as a possible normalizing effect of antidepressant treatment. The paper by Abbott et al. (2013) suggested that patients with MDD present a hyperconnectivity pattern in the posterior areas of DMN, PFDLC, and $\mathrm{dmCPF}$, which tends to recover after the treatment. The paper by Wu et al. (2011) showed a mixed pattern of hypoconnectivity in the posterior areas of DMN, and hyperconnectivity in the most anterior areas of the same network at rest in depressed patients. In the same paper, hypoconnectivity between PCC and sgACC recovered, but not PCC-dmPFC hyperconnectivity (Wu et al., 2011). The paper by Beall et al. (2012), with a pre- and posttreatment design, showed an increase in PCC-ACC connectivity after ECT treatment. Four studies investigated the possible correlation between clinical improvement of depression and changes in connectivity (Beall et al., 2012; Abbott et al., 2013; Li et al., 2013; Posner et al., 2013). Only the study by Beall et al. (2012) found that clinical improvement correlated with changes in ACC-DLPFC connectivity. The other papers found no correlations between clinical symptom improvement and changes in connectivity (Abbott et al., 2013; Li et al., 2013; Posner et al., 2013). The specific results from each study have been summarized in Tables 3A,B, 4A,B.

Six studies approached the changes in connectivity after depression treatment in cortical-limbic connectivity, one of them conducted at rest (Salomons et al., 2014), and the others using several affective tasks (Anand et al., 2005; Chen et al., 2008; Lisiecka et al., 2011; Beall et al., 2012; Heller et al., 2013). Two of them compared the connectivity between ACC, frontal and limbic regions of depressed patients with control patients at the baseline measure. Both presented similar results. On the one hand, the one by Anand et al. (2005) found hypoconnectivity between ACC and the subcortical structures such as the medial thalamus and the pallidostriatum. On the other, Chen et al. (2008) found a pattern of hypoconnectivity between the amygdala and several cortical and subcortical structures (Chen et al., 2008). Likewise, both papers found important increases in connectivity after the treatment. In the paper by Anand et al. (2005) no differences in connectivity were found after the treatment between the patients and the control subjects at rest and when exposed to negative stimuli (visual images with negative affective valence from "The International Affective Picture System"; IAPS). It has also been suggested that the amygdala connectivity becomes normalized after antidepressive treatment (Chen et al., 2008).The paper by Lisiecka et al. (2011) compared the changes in brain connectivity between two groups of depressed patients, one treated with venlafaxine and the other with mirtazapine. After the treatment, the patients presented increases in connectivity between dmPFC and cerebellum, cingulated cortex, parietal cortex, and decreases in connectivity between the OFC and the right medial cingulated cortex, the middle temporal gyrus, the superior occipital gyrus, the right fusiform gyrus, and the inferior temporal gyrus. Also the same paper showed decreases in connectivity between left the OFC and the left superior parietal gyrus, the pre-cuneus, and the post-central gyrus, the left medial temporal gyrus, the cuneus, the calcarine fissure, and the angular gyrus (Lisiecka et al., 
2011). This study also suggested that venlafaxine changed the OFC-cerebellum coupling, whereas mirtazapine increased the OFC-DLPFC connectivity. Three studies measured changes in brain connectivity after antidepressant treatment in a pre- and post-treatment design (Beall et al., 2012; Heller et al., 2013; Salomons et al., 2014). The work by Beall et al. (2012) found a pattern of increases and decreases in connectivity between ACC, the caudate nucleus and frontal and parietal cortical areas. The work by Salomons et al. (2014) showed-among other findingsdecreases in connectivity between $\mathrm{dmPFC}$ and some limbic structures. The work by Heller et al. (2013) found connectivity between the nucleus accumbens, MFG and accumbens-VMPFC related to changes in affection. Out of all these papers, four of them (Anand et al., 2007; Beall et al., 2012; Heller et al., 2013; Salomons et al., 2014) conducted analyses of the correlations between connectivity changes and the improvement of depressive symptoms measured through clinical scales (Anand et al., 2007; Beall et al., 2012; Salomons et al., 2014), or correlations between changes in connectivity and specific symptoms improvement, such as alterations of affection or anhedonia (Heller et al., 2013). The four papers found a positive link between both types of variables. Each paper's specific results have been summarized in Tables 3A,B, 4A,B.

Three papers studied changes in connectivity in CCN after depression treatment (Aizenstein et al., 2009; Perrin et al., 2012; Liston et al., 2014). In the first paper, the authors found that depressed patients showed less connectivity between DLPFC and dACC than control subjects (Aizenstein et al., 2009). The paper by Liston et al. (2014) found hypoconnectivity in depressed patients between DLPFC and MFG (BA 46), posterior parietal areas, and other prefrontal areas (Liston et al., 2014). In none of the studies was connectivity recovered after depression treatment (Aizenstein et al., 2009; Liston et al., 2014), although the study by Liston et al. (2014) did find improvement after treatment in DMN. The paper by Perrin et al. (2012) suggested that patients experienced reductions in connectivity related to the treatment within 1_DLPFC and the prefrontal cortex (BA 44, 45 , and 46) as well as reductions in connectivity between ACC and areas of OFC and r-DLPFC among others (Perrin et al., 2012). Each paper's specific results have been summarized in Tables 3A,B, 4A,B.

Lastly, six papers could not be classified into the above division. The paper by Abbott et al. (2014) suggested that depressed patients show hypoconnectivity of each hippocampus with the contralateral medial temporal lobe and of $r_{-}$HPC with ACC. In this study, after the treatment, only the right hippocampus connectivity increased, which the authors attribute to the hemisphere where ECT was applied (Abbott et al., 2014). Moderate correlations were reported in this paper between connectivity recovery and clinical improvement. On the other hand, the paper by Wei et al. (2014) suggested that depressed patients present hypoconnectivity in the Voxel Mirrored Homotopic Connectivity between both MFG (BA 8/9) and both angular gyri (BA 49). The paper also suggested that, after treatment, the connectivity between both hemispheres increases and tends to become normalized (Wei et al., 2014).The paper by Wang et al. (2014) suggested that depressed patients show smaller Functional Connectivity Strength maps (FCS) than healthy subjects in both hippocampi, and the opposite pattern in the dmPFC cortexes of both hemispheres. Antidepressant treatment tended to reverse the pattern so that, after treatment, FCS increased between both hippocampi and it decreased between dmPFCs. Additionally, the changes in $\mathrm{dmPFC}$ connectivity correlated positively with the clinical improvement measures (Wang et al., 2014). In the paper by Yang et al. (2014), we observed increases in the connectivity of the hypothalamus with temporal areas and of the basal ganglia, and decreases of the connectivity of the hypothalamus with frontal and medial areas, the thalamus, and the cerebellum (Yang et al., 2014). The work by Baeken et al. (2014) found that the successful depression treatment with high frequency rTMS was associated to an increase in connectivity between sgACC and perigenual ACC, both seeds of either DMN and AN. Each paper's specific results are summarized in Tables 3A,B, 4A,B.

\section{DISCUSSION}

The goal of the current paper is to review the studies investigating the effect of antidepressant treatment on brain connectivity. Some conclusions may be extracted from the current review. Firstly, out of the 19 papers reviewed, all but one (Aizenstein et al., 2009) reported that, after depression treatment and the corresponding improvement, there occur changes in brain connectivity. As can be observed in Tables 1, 2, despite the fact that most of the studies were conducted on patients with MDD (Anand et al., 2005; Chen et al., 2008; Lisiecka et al., 2011; Beall et al., 2012; Abbott et al., 2013, 2014; Heller et al., 2013; Li et al., 2013; Liston et al., 2014; Salomons et al., 2014; Wang et al., 2014), depression treatment is also related to changes in connectivity in patients with LOD (Wu et al., 2011; Andreescu et al., 2013), in dysthymic patients (Posner et al., 2013), and in more heterogenic samples of patients with unipolar depression (Perrin et al., 2012; Wei et al., 2014; Yang et al., 2014). Likewise, several of these papers include, among their samples, treatment-resistant patients, or ones with comorbid anxiety disorders among other clinical characteristics. Accordingly, successful treatment of unipolar depression seems to be generally related to changes in brain connectivity.

As regards the connectivity networks studied in the current paper, the results summarized in Tables 3A,B, 4A,B allow us to conclude that both depression and depression improvement are related to changes in connectivity in DMN as all the papers included in this review find this association positive (Wu et al., 2011; Beall et al., 2012; Abbott et al., 2013; Andreescu et al., 2013; Li et al., 2013; Posner et al., 2013; Liston et al., 2014). Most of these papers found basal a hyperconnectivity pattern in the DMN of depressed patients-already discussed in the Results Sectionexcept for the paper by Abbott et al. (2014), where a different pattern was found. This hyperconnectivity pattern in DMN is to be expected given that-as discussed in the introduction-it is a common finding in most studies and was confirmed in a recent meta-analysis (Kaiser et al., 2015). It is an interesting fact 
that all the studies reviewed found changes in DMN connectivity after treatment, and most studies found that DMN connectivity tends to normalize or resemble more that of healthy subjects after treatment (Wu et al., 2011; Abbott et al., 2013; Andreescu et al., 2013; Li et al., 2013; Posner et al., 2013; Liston et al., 2014). However, most studies-except for Abbott et al. (2013) maybesuggested that the normalization of DMN connectivity after treatment is incomplete. In the paper by Andreescu et al. (2013), the antidepressant treatment was associated to an increased connectivity in the anterior frontal nodes of the DMN, but the alterations of PCC connectivity found in their depressed patients prior to treatment was not recovered. In the paper by Liston et al. (2014), the treatment was associated to a decrease in sgACC connectivity with other DMN structures, but the connectivity of pgACC with the thalamus and the pre-cuneus did not change.

Something similar may be concluded from the studies measuring connectivity changes between cortical-limbic structures (Anand et al., 2005; Chen et al., 2008; Lisiecka et al., 2011; Beall et al., 2012; Heller et al., 2013; Salomons et al., 2014). In fact, in all these papers, the patients showed changes in connectivity between different cortical and limbic structures after depression treatment. According to the emotional valence of the stimulus, the papers by Anand et al. (2005) and Heller et al. (2009) showed similar results given that, in both papers, structures that are critical to emotional processing such as ACC and AMY present hypoconnectivity in depressed patients when compared to healthy ones in situations of negative emotions processing. More importantly, their data suggests that antidepressant treatment tends to normalize the connectivity in these structures (Anand et al., 2005; Chen et al., 2008). The paper by Beall et al. (2012) showed that ECT treatment diminished the activity of OFC when faced with negative valence stimuli. Nevertheless, the connectivity analysis of this paper was made only for the rest condition, and the authors found the hyper and hypoconnectivity mixed pattern of the OFC that is described in Table 4B (Beall et al., 2012), which is difficult to interpret due to the methodology used in that study. The paper by Heller et al. (2013) focuses on positive emotional processing. A previous study not included in this meta-analysis-(Heller et al., 2009)showed that depressed patients had difficulty in sustaining the connectivity between the nucleus accumbens and the DLPFC when they were instructed to enhance positive emotions elicited by positive images. In the paper included in our review, its data suggests that antidepressant treatments improved sustained connectivity between the nucleus accumbens and vmPFC when patients performed the same kind of task, and these gains were related to gains in positive affect after treatment (Heller et al., 2013). The work by Lisiecka et al. (2011) is difficult to discuss in terms of the emotional valence of the stimulus because the authors performed an OFC seed-whole brain connectivity analysis and found a very complex pattern of OFC connectivity changes that is hardly interpretable in terms of functional connectivity related to the emotional valence processing. Finally the paper by Salomons et al. (2014) showed that a successful treatment was associated with increased dmPFC-thalamic connectivity and decreased sgACC-caudate connectivity. Although performed at rest, it should be noted that, in their study, these changes strongly correlated with improvements in depressive symptoms. In other studies, sgACC connectivity with striatal regions has been related to the maladaptive emotion regulation of depressed patients (Furman et al., 2011). Therefore, the connectivity changes in the paper by Salomons et al. (2014) were possibly somewhat related to changes in positive or negative emotional processing. Taken together, the results of our review resembles to some extent those of Ma (2014), because successful antidepressant treatment may be associated to both activation and connectivity changes in different emotional subnetworks during emotional negative processing, and probably also during positive emotional experiences. However, the number of connectivity studies performed to date is too small to establish the specific connectivity modifications after depression treatment.

Nonetheless, it is not clear that the same thing happens with the connectivity of the Cognitive Network structures since, out of the three papers studying this network (Aizenstein et al., 2009; Perrin et al., 2012; Liston et al., 2014), only one reports a positive relationship between connectivity changes and depression improvement (Perrin et al., 2012), whereas longitudinal-case control studies show more permanent alterations in this network. Although by no means does this allow us to conclude that CCN is a treatment-resistant network, it is noteworthy that it is the only network yielding this type of result. It is even more noteworthy if we consider that, in addition to the papers on DMN and cortical-limbic connections, there exist reports on the positive relationship between depression improvement and connectivity changes in the hippocampus (Abbott et al., 2013), the hypothalamus (Yang et al., 2014), between both brain hemispheres (Wei et al., 2014), between sgACC and perigenual ACC (Baeken et al., 2014), and in studies conducted with connectivity analyses of the whole brain (Wang et al., 2014). Undoubtedly, further studies are needed to find out what happens to CCN after treatment.

We find positive correlations between clinical improvement and changes in brain connectivity in three papers investigating cortical-limbic connectivity changes (Anand et al., 2007; Heller et al., 2013; Salomons et al., 2014): one investigates connectivity changes between HPC and dmPFC and the rest of the brain (Wang et al., 2014); and then the paper by Beall et al. (2012), which was conducted with seeds belonging to DMN and the limbic system. However, the papers investigating the relationship between depression treatment and connectivity changes in DMN found no such association (Abbott et al., 2013; Li et al., 2013; Posner et al., 2013). Taken together, the data suggest the possibility of a relationship between recovery from depression symptoms and recovery of connectivity between several cortical-limbic structures. The close relationship between clinical recovery and connectivity changes between these brain areas may be somewhat expectable because the limbic system is closely related to the mood, anxiety, and vegetative symptoms that constitute the core depressive symptoms. However, the lack of a relationship between clinical improvement and changes in connectivity in DMN might be expectable given that DMN would be related to rumination but not to core 
depression symptomatology as indexed in the HAM-D scale (Posner et al., 2013). Interestingly, the paper by Beall et al. (2012) found a correlation between depression symptoms improvement and rACC-rDLPFC connectivity. Given that other studies measuring connectivity between other networks also find this type of correlation (Abbott et al., 2014; Wang et al., 2014), this phenomenon should be studied in more depth.

Finally, we would like to mention that some papers hint at the existence of a causal relationship between recovery from depression symptoms and specific changes in brain connectivity. For example, the paper by Salomons et al. (2014) — where rTMS sessions were applied on $\mathrm{dmPFC}$ as a depression treatmentfound post-treatment changes between $\mathrm{dmPFC}$ and other limbic structures. The paper by Abbott et al. (2014) found posttreatment changes in connectivity in the right hemisphere, which is attributed to the fact that the ECT sessions were mostly applied on the right side and unilaterally (Abbott et al., 2014). Interestingly, the paper by Liston et al. (2014) applied rMTS sessions to DLPFC but found no changes in connectivity in $\mathrm{CCN}$. The paper by Heller et al. shows that treatment with duloxetine was related to changes in connectivity in DMN, but the same thing did not happen with the placebo group. Some papers suggest that some specific changes in connectivity after depression treatment might depend, partly, on the specific pharmacological treatment administered (Lisiecka et al., 2011), although the paper by Heller et al. (2013) found no differences between treatment groups in the correlation between changes in the connectivity of BA 46 and the positive affection improvement among its depressed patients. Therefore, the selection of papers suggests that part of the changes in brain connectivity experienced after depression treatment might depend-partly, at least-on the mechanisms of action of the treatment administered. This, in turn, would not only bear important clinical implications, but it would also open the door for the use of connectivity as a tool to study the mechanisms of action and therapeutic of several depression treatments.

The current paper has some limitations, too, namely the fact that it is a systematic review but not a meta-analysis. The number of studies and their heterogeneity advised us against the latter type of study. In addition, the number of papers that can be included in the review, the variety in research designs, and the variety of treatments administered among other variables render it very difficult to build a conclusive model on brain connectivity related to depression treatment. Therefore, we are unable to build an integrative model about the changes in brain connectivity after depression treatment. For these reasons, the conclusions of this paper should be considered as preliminary. Another limitation of the present work is the publication bias that usually occurs in fMRI studies (Jennings and Van Horn, 2012). In fact, disregarding reports as that from Aizenstein et al. (2009), researches in which positive association between successful antidepressant treatment and changes in brain connectivity could not be demonstrated might remain unpublished. Indeed, this and other relevant aspects could bias our review. Nevertheless, the paper also has some strength. To our knowledge, it is the only paper to review systematically treatment-related changes in brain connectivity measured through fMRI, and we can offer a few preliminary conclusions on the relationship between the treatment of depression and changes in brain connectivity. They are listed below.

The somatic treatment of depressive symptoms seems to be associated generally to changes in brain connectivity. As for DMN, in most of the studies included, it was found that this network is hyperactive in depressed patients, and that the treatment tends to normalize DMN hyperconnectivity. However, this normalization is usually incomplete, and the frontal portions of DMN might probably still present alterations of connectivity after treatment. The connectivity of corticallimbic connections related to the emotional processing also changes after depression treatment. The studies suggest that a successful antidepressant treatment would be linked to changes in the connectivity of different sub-networks involved in negative emotional processing, and possibly also in the processing of positive emotions. Current data suggests that CCN might not undergo changes after a successful antidepressive treatment. In a rather consistent way, changes in connectivity between cortical-limbic structures correlate with an improvement of the core depressive symptoms. However, there seems to be no link between changes in the connectivity of DMN after antidepressive symptom and the improvement of the clinical symptoms of the condition. Lastly, we should point out that some papers hint at the existence of a causal relationship between recovery from depression symptoms and specific changes in brain connectivity, and also that depression treatment might depend-partly, at least-on the mechanisms of action of the treatment administered, which would have important theoretical and clinical implications.

Another strength of our work is the fact that it allows us to make a series of recommendations that, on the one hand, it might guide future researchers exploring the effect of anti-depression treatments on brain connectivity and, on the other, they would help to report some of the data of this type of study to improve its scientific quality.

1. Presenting complete sociodemographic data: As can be seen in Tables 1, 2, in various papers, these data are not sufficiently reported. However, they are essential to understand the nature of the sample on which the study was conducted.

2. Reporting the data related to the psychopathology and the psychopathological evaluation of the patients: Some of the studies comprised in the current review only mention that they had a depression, but the types of the patients' depressive disorders are not specified, nor are the criteria under which they were diagnosed. In several papers, the data on the severity of depressive symptomatology are incomplete. Sometimes even essential data are missing, such as the standard deviation of the Hamilton scale scores, before and after the treatment. In addition, many papers do not report data on the possible psychopathological comorbidities of the depressed patients. It might be advisable to present all these data in the Results Section of the articles for a better comprehension of the type of sample used in each study. 
3. Increasing the types of clinical instruments used: In most of the studies reviewed, the effect of anti-depression treatment is measured through the Hamilton scale for depression and, when trying to establish correlations among changes in brain connectivity after antidepressant treatment, they are often non-significant. However, this does not necessarily mean that there is no relevant association between the clinical improvement of specific symptoms of depression and brain connectivity. For example, clinical scales such as the Beck scale, which measures symptoms of rumination among others, might show correlations between the possible changes in DMN after the treatment and improvement of clinical symptomatology. The study by Heller et al. (2013) found correlations between changes in brain connectivity and improvement of positive and negative affect. Nevertheless, despite the fact that depression is an affective disorder, affection scales are not commonly used to monitor clinical change in depressed patients. These instruments along with others-such as, for example, neuropsychological tests that measure specific cognitive domains-should be used if we mean to establish correlations between changes in connectivity and clinical improvement.

4. Conducting, as far as possible, hypothesis-driven studies: In the current review we can observe a few papers with datadriven analyses, or where the correlations of one ROI with the full brain were studied. The problem with these studies is that data interpretation is complex and, logically, the authors tend to discuss the part of the results most relevant to them, while discarding another part of the results as possible "noise." The problem with this approach is that, as we mentioned in the introduction, and as can be seen in Tables 3, 4, the brain areas affected by depression are very diverse and, therefore, this type of study runs the risk of not interpreting or not giving relevance to changes in certain connectivity patterns after the treatment of depression that might be important.

5. Using a control group to better establish the magnitude of the change in connectivity. Pre- and post-studies allow us to see whether there are changes in brain connectivity after antidepressant treatment. However, in many of the studies, there is no control group, which prevents us from knowing the magnitude of alterations in brain connectivity and, therefore, we cannot know whether brain connectivity-or to what extent changes in brain connectivity after antidepressant treatment-shows a tendency toward the recovery of the connectivity of a healthy person. We should also bear in mind that pre- and post-treatment designs tend to overestimate the magnitude of connectivity changes after treatment. Accordingly, the lack of a control group somewhat hinders the interpretability of the data in terms of an effect size.

6. Using research designs that allow us to establish cause-effect relationships: As we mentioned above, some studies suggest the existence of a causal relationship between the treatment of depression and changes in brain connectivity. However, pre- and post-treatment designs are not the most suitable to establish this type of relationship, given the lack of adequate methodological control measures. It is suggested that, as far as possible, these studies should compare treatments among themselves, or else that short studies should be designed with sham or placebo groups. However, the latter may be difficult, as it would involve leaving a group of patients untreated, which would be disputable from an ethical point of view because these papers do not study the possible efficacy of the treatments.

We are aware that the latter two points are, to some extent, incompatible. Establishing a brain connectivity baseline and trying to establish causal relationships between treatment and changes in connectivity would involve, most of the time, designing studies with more than two groups and their corresponding follow-up. However, we do believe that, in designing future studies, they should ideally either comprise a control group, or choose a design that allows us to better research the possible causal relationships between the effect of treatment and changes in brain connectivity, like using two arms of active treatment. The current review allows us to establish that, generally, the treatment of depression is linked to changes in brain connectivity. The two most relevant questions to answer at the moment would be, on the one hand, whether antidepressant treatment is linked to the recovery of normal connectivity and to what extent, and on the other, whether there is a cause-effect relationship between the treatment of depression and the recovery of connectivity, or else if the link between both phenomena is influenced by other variables. The current state of the art does not allow us to identify bigger matters or venture more complex hypotheses on this topic. This paper clearly shows how, at the moment, a descriptive one is the best methodological approach to review the contributions of the estimations of brain connectivity models in the study of the effectiveness of treatments related to MDD. This field is no different from other similar ones, both medical and psychological. For example, the situation resembles-to a great extent - that which is perceived in disorders unrelated to the one discussed here. For instance, papers on fMRI in type 1 diabetes (Gallardo et al., 2015) show the same embryonic state as the brain connectivity effects of depression treatment, we have noted. Accordingly, the current state of affairs should be considered, in our opinion, an undeniably relevant opportunity for scientific breakthrough.

\section{ACKNOWLEDGMENTS}

This study was supported by the Grup de Recerca en Tècniques Estadístiques Avançades Aplicades a la Psicologia (GTEAAP) members of the Generalitat de Catalunya's 2014 SGR 326 Consolidated Research Group (GRC) and was made possible by the PSI2013-41400-P project of Ministerio de Economia $y$ Competitividad of the Spanish Government. The work was also partially supported by Programa de Investigación 2014-2015 de la Coordinación de la Investigación Científica de la Universidad Michoacana de San Nicolás de Hidalgo. 


\section{REFERENCES}

*Abbott, C. C., Jones, T., Lemke, N. T., Gallegos, P., McClintock, S. M., Mayer, A. R., et al. (2014). Hippoccampal structural and functional changes associated with electroconvulsive theraphy response. Transl. Psychiatry 4, 1-7. doi: 10. 1038./tp. 2014.124

*Abbott, C. C., Lemke, N. T., Gopal, S., Thoma, R. J., Bustillo, J., Calhoun, V. D., et al. (2013). Electroconvulsive therapy response in major depressive disorder: a pilot functional network connectivity resting state FMRI investigation. Front. Psychiatry 4:10. doi: 10.3389/fpsyt.2013.00010

*Aizenstein, H. J., Butters, M. A., Wu, M., Mazurkewicz, L. M., Stenger, V. A., Gianaros, P. J., et al. (2009). Altered functioning of the executive control circuit in late-life depression: episodic and persistent phenomena. Am. J. Geriatr. Psychiatry 17, 30-42. doi: 10.1097/JGP.0b013e31817b60af

Anand, A., Li, Y., Wang, Y., Gardner, K., and Lowe, M. J. (2007). Reciprocal effects of antidepressant treatment on activity and connectivity of the mood regulating circuit: and FMRI study. J. Neuropsychiatry Clin. Neurosci. 19, 274-282. doi: 10.1176/appi.neuropsych.19.3.274

*Anand, A., Li, Y., Wang, Y., Wu, J., Gao, S., Bukhari, L., et al. (2005). Antidepressant effect on connectivity of the mood-regulating circuit: an FMRi study. Neuropsychopharmacology 30, 1334-1334. doi: 10.1038/sj.npp.1300725

*Andreescu, C., Tudorascu, D. L., Butters, M. A., Tamburo, E., Patel, M., Price, J., et al. (2013). Resting-state functional connectivity and treatment-response in late-life depression. Psychiatry Res. 214, 313-321. doi: 10.1016/j.pscychresns.2013.08.007

*Baeken, C., Marinazzo, D., Wu, G. R., Van Schuerbeek, P., De Mey, J., Marchetti, I., et al. (2014). Accelerated HF-rTMS in treatment -resistant unipolar depression: insights from subgenual anterior cingulate functional connectivity. World J. Biol. Psychiatry 15, 286-297. doi: 10.3109/15622975.2013.872295

*Beall, E. B., Malone, D. A., Dale, R. M., Muzina, D. J., Koenig, K. A., Bhattacharrya, P. K., et al. (2012). Effeects of electroconvulsive therapy on brain functional activation and connectivity in depression. J. ECT 28, 234-241. doi: 10.1097/YCT.0b013e31825ebcc7

Belleau, E. L. L., Taubitz, L. E., and Larson, C. L. (2014). Imbalance of default mode and regulatory networks during externally focused processing in depression. Soc. Cogn. Affect. Neurosci. 10, 744-751. doi: 10.1093/scan/nsu117

Broyd, S. J., Demanuele, C., Debener, S., Helps, S. K., James, C. J., and Sonuga-Barke, E. J. (2009). Default-mode brain dysfunction in mental disorders: a systematic review. Neurosci. Biobehav. Rev. 33, 279-296. doi: 10.1016/j.neubiorev.2008.09.002

*Chen, C. H., Suckling, J. O. C., Fu, C. H., Williams, S. C. R., Walsh, N. D., Mitterschiffthaler, M. T., et al. (2008). Functional coupling of the amygdale in depressed patients treated with antidepressant medication. Neuropsychopharmacology 33, 1909-1918. doi: 10.1038/sj.npp.1301593

Corbetta, M., and Shulman, G. L. (2002). Control of goal-directed and stimulus-driven attention in the brain. Nat. Rev. Neurosci. 3, 201-2015. doi: 10.1038/nrn755

Delaveau, P., Jabourian, M., Lemogne, C., Guionnet, S., Bergouignan, L., and Fossati, P. (2011). Brain effects of antidepressants in major depression: a metaanalysis of emotional processing studies. J. Affect. Disord. 130, 66-74. doi: 10.1016/jad.2010.09.032

Dutta, A., McKie, S., and Deakin, J. F. W. (2014). Resting-state networks in major depressive disorder. Psychiatry Res. 224, 139-151. doi: 10.1016/j.pscychresns.2014.10.003

Fales, C. L., Barch, D. M., Rundle, M. M., Mintun, M. A., Snyder, A. Z., Cohen, J. D., et al. (2008). Altered emotional interference processing in affective and cognitive-control brain circuitry in major depression. Biol. Psychiatry 63, 377-384. doi: 10.1016/j.biopsych.2007.06.012

Fitzgerald, P. B., Laird, A. R., Maller, J., and Daskalakis, Z. J. (2014). A metaanalytic study of changes I brain activation in depression. Hum. Brain. Mapp. 29, 683-695. doi: 10.1002/hbm.20426

Friston, K. J. (2011). Functional and effective connectivity. A review. Brain Connect. 1, 13-30. doi: 10.1089/brain.2011.0008

Furman, D. J., Hamilton, J. P., and Gotlib, I. H. (2011). Frontostriatal functional connectivity in major depressive disorder. Biol. Mood Anxiety Disord. 1:11. doi: 10.1186/2045-5380-1-11

Gallardo, G., González-Garrido, A. A., Gudayol-Ferré, E., and Guàrdia-Olmos, J. (2015). Type 1 Diabetes modifies brain activation in young patients while performing visuospatial working memory tasks. J. Diabetes Res. 2015:703512. doi: 10.1155/2015/703512

Greicius, M. D., Flores, B. H., Menon, V., Glover, G. H., Solvason, H. B., Kenna, H., et al. (2007). Resting-state functional connectivity in major depression: abnormally increased contributions from subgenual cingulate cortex and thalamus. Biol. psychiatry 62, 429-437. doi: 10.1016/j.biopsych.2006. 09.020

Greicius, M. D., Krasnow, B., Reiss, A. L., and Menod, V. (2003). Functional connectivity in the resting brain: a network analysis of the default mode hypothesis. Proc. Natl. Acad. Sci. U.S.A. 100, 253-258. doi: 10.1073/pnas.0135058100

Greicius, M. D., Supekar, K., Menon, V., and Dougherty, R. F. (2009). Restingstate functional connectivity reflects structural connectivity in the default mode network. Cereb. cortex 19, 72-78. doi: 10.1093/cercor/bhn059

Guo, W., Liu, F., Xue, Z., Gao, K., Liu, Z., Xiao, C., et al. (2013). Abnormal resting-state cerebellar-cerebral functional connectivity in treatment-resistant depression and treatment sensitive depression. Progr. Neuropsychopharmacol. Biol. Psychiatry 44, 51-57. doi: 10.1016/j.pnpbp.2013.01.010

Heller, A. S., Johnstone, T., Light, S. N., Peterson, M. J., Kolden, G. G., Kalin, N. H., et al. (2013). Relationships between changes in sustained fronto-striatal connectivity and positive affect in major depression resulting from antidepressant treatment. Am. J. Psychiatry 170, 197-206. doi: 10.1176/appi.ajp.2012.12010014

*Heller, A. S., Johnstone, T., Shackman, A. J., Light, S. N., Peterson, M. J., Kolden, G. G., et al. (2009). Reduced capacity to sustain positive emotion in major depression reflects diminished maintenance of front o-striatal brain activation. Proc. Natl. Acad. Sci. U.S.A. 106, 22445-22245. doi: 10.1073/pnas.09106 51106

Jacobs, R. H., Jenkins, L. M., Gabriel, L. B., Barba, A., Ryan, K. A., Weisenbach, S. L., et al. (2014). Increased coupling of intrinsic networks in remitted depressed youth predicts rumination and cognitive control. PLoS ONE 9:e104366. doi: 10.1371/journal.pone.0104366

Jennings, R. G., and Van Horn, J. D. (2012). Publication bias in neuroimaging research: implications for meta-analyses. Neuroinformatics 10, 67-80. doi: 10.1007/s12021-011-9125-y

Kaiser, R. H., Andrews-Hanna, J. R., Wager, T. D., and Pizzagalli, D. A. (2015). Large-scale network dysfunction in major depressive disorder. JAMA Psychiatry 72, 603-611. doi: 10.1001/jamapsychiatry.2015.0071

*Li, B., Liu, L., Friston, K. J., Shen, H., Wang, L., Zeng, L. L., et al. (2013). A treatment- resistant default mode subnetwork in major depression. Biol. Psychiatry 74, 48-54. doi: 10.1016/j.biopsych.2012.11.007

*Lisiecka, D., Meisenzahl, E., Scheurecker, J., Schoepf, V., Withhy, P., Chaney, A., et al. (2011). Neural correlates of treatment outcome in masjor depression. Int. J. Neuropsychopharmacol. 14, 521-534. doi: 10.1017/S1461145710001513

*Liston, C., Chen, A. C., Zebley, B. D., Drysdale, A. T., Gordon, R. Leuchter, B., et al. (2014). Default mode network mechanisms of transcranial magnetic stimulation in depression. Biol. Psychiatry 7, 517-526. doi: 10.1016/j.biopsych.2014.01.023

Ma, Y. (2014). Neuropsychological mechanism underlying antidepressant effect: a systematic meta-analysis. Mol. Psychiatry 20, 311-319. doi: 10.1038/mp.2014.24

*Perrin, J. S., Merz, S., Bennett, D. M., Currie, J., Steele, D. J., Reid, I. C., et al. (2012). Electroconvulsive therapy reduces frontal cortical connectivity in severe depressive disorder. Proc. Natl. Accad. Sci. U.S.A. 109, 5464-5468. doi: 10.1073/pnas.1117206109

* Posner, J., Hellerstein, D. J., Gat, I., Mechling, A., Klahr, K., Wang, Z., et al. (2013). Antidepressants normalize the default mode network in patients with dysthymia. JAMA Psychiatry 70, 373-382. doi: 10.1001/jamapsychiatry.2013.455

Raichle, M. E., McLeod, A. M., Snyder, A. Z., Powers, W. J., Gusnard, D. A., and Shulman, G. L. (2001). A default mode of brain function. Proc. Natl. Acad. Sci. U.S.A. 98, 676-682. doi: 10.1073/pnas.98.2.676

Rogers, M. A., Kasa, I. K., Matsuo, K., Fukuda, R., Iwanami, A., Nakagome, K., et al. (2004). Executive and prefrontal dysfunction in unipolar depression: a review of neuropsychological and imaging evidence. Neurosci. Res. 50, 1-11. doi: 10.1016/j.neures.2004.05.003

Rose, E. J., Simonotto, E., and Ebmeier, K. P. (2006). Limbic over-activity in depression during preserved performance on the n-back task. Neuroimage 29, 203-215. doi: 10.1016/j.neuroimage.2005.07.002 
*Salomons, T. V., Dunlop, K., Kennedy, S. H., Flint, A., Geraci, J., Giacobbe, P., et al. (2014). Resting-state cortico-thalamic-striatal connectivity predicts response to dorsomedial prefrontal rTMS in major depressive disorder. Neuropsychophamacology 39, 488-498. doi: 10.1038/npp.2013.222

Sheline, Y. I., Price, J. L., Yan, Z., and Mintun, M. A. (2010). Restingstate functional MRI in depression unmasks increased connectivity between networks via the dorsal nexus. Proc. Natl. Acad. Sci. U.S.A. 107, 11020-11025. doi: $10.1073 /$ pnas. 1000446107

Siegle, G. J., Thompson, W., Carter, C. S., Steinhauer, S. R., and Thase, M. E. (2007). Increased amygdala and decreased dorsolateral prefrontal BOLD responses in unipolar depression: related and independent features. Biol. Psychiatry 61, 198-209. doi: 10.1016/j.biopsych.2006.05.048

Thomas, E. J., and Elliott, R. (2009). Brain imaging correlates of cognitive impairment in depression. Front. Hum. Neurosci. 3:30. doi: 10.3389/neuro.09. 030.2009

Veer, I. M., Beckmann, C. F., van Tol, M. J., Ferrarini, L., Milles, J., Veltman, D. J., et al. (2010). Whole brain resting-state analysis reveals decreased functional connectivity in major depression. Front. Syst. Neurosci. 4:41. doi: $10.3389 /$ fnsys. 2010.00041

*Wang, L., Hermens, D. F., Hickie, I. B., and Lagopoulos, J. (2012). A systematic review of resting-state functional-MRI studies in major depresion. J. Affect. Disord. 142, 6-12. doi: 10.1016/j.jad.2012.04.013

Wang, L., Xia, M., Li, K., Zeng, Y., Su, Y., Dai, W., et al. (2014). The effects of antidepressant treatment on resting-state functional brain networks in patients with major depressive disorder. Hum. Brain Mapp. 36, 768-778. doi: 10.1002./hbm.22663
*Wei, Q., Tian, Y., Yu, Y., Zhang, F., Hu, X., Dong, Y., et al. (2014). Modulation of interhemisferic functional coordination in electroconvulsive therapy. Transl. Psychiatry 4, 1-8. doi: 10.1038/tp.2014.101

Wise, T., Cleare, A. J., Herane, A., Young, A. H., and Arnone, D. (2014) Diagnostic and therapeutic utility of neuroimaging in depression: an overview. Neuropsychiatr. Dis. Trat. 10, 1509-1518. doi: 10.2147/NDT.S50156

*Wu, M., Andreescu, C., Butters, M. A., Tamburo, R., Reynolds, C. F. III, and Aizenstein, H. (2011). Default-mode network connectivity and with matter burden in late-life depression. Psychiatry Res. 194, 39-46. doi: 10.1016/j.pscychresns.2011.04.003

*Yang, R., Zhang, H., Wu, X., Yang, J., Ma, M., Gao, Y., et al. (2014). Hypothalamus-anchored resting brain network changes before and after sertraline treatment in major depression. Biomed. Res. Int. 2014:915026. doi: $10.1155 / 2014 / 915026$

Conflict of Interest Statement: The authors declare that the research was conducted in the absence of any commercial or financial relationships that could be construed as a potential conflict of interest.

Copyright (C) 2015 Gudayol-Ferré, Peró-Cebollero, González-Garrido and GuàrdiaOlmos. This is an open-access article distributed under the terms of the Creative Commons Attribution License (CC BY). The use, distribution or reproduction in other forums is permitted, provided the original author(s) or licensor are credited and that the original publication in this journal is cited, in accordance with accepted academic practice. No use, distribution or reproduction is permitted which does not comply with these terms. 